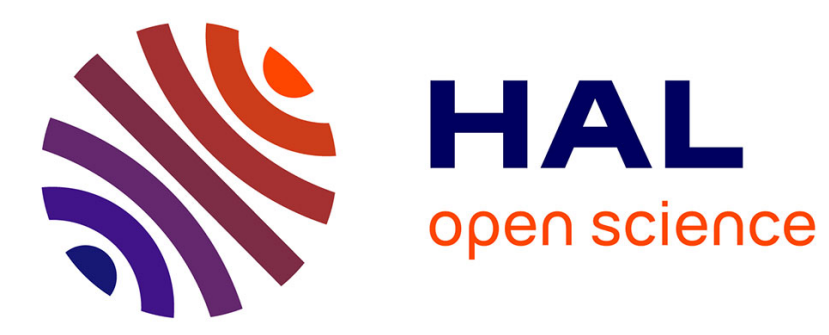

\title{
Les questions de temporalité dans les méthodes de recherche en didactiques.
}

Dominique Lahanier-Reuter, Eric Roditi

\section{To cite this version:}

Dominique Lahanier-Reuter, Eric Roditi. Les questions de temporalité dans les méthodes de recherche en didactiques.. Dominique Lahanier-Reuter et Eric Roditi. Questions de temporalité., Presses Universitaires du Septentrion, pp.9-13, 2007, Education et didactiques. halshs-00349774

\section{HAL Id: halshs-00349774 \\ https://shs.hal.science/halshs-00349774}

Submitted on 19 Jul 2011

HAL is a multi-disciplinary open access archive for the deposit and dissemination of scientific research documents, whether they are published or not. The documents may come from teaching and research institutions in France or abroad, or from public or private research centers.
L'archive ouverte pluridisciplinaire HAL, est destinée au dépôt et à la diffusion de documents scientifiques de niveau recherche, publiés ou non, émanant des établissements d'enseignement et de recherche français ou étrangers, des laboratoires publics ou privés. 


\section{Présentation}

\section{Lahanier-Reuter Dominique, Université Lille 3, Équipe THEODILE Roditi Éric, Université Paris 5, Équipe EDA}

Le présent volume est le deuxième de la collection "éducation et didactiques" qui soit entièrement consacré aux méthodes de recherche en didactiques. Le premier volume a traité trois axes de questionnement méthodologique: la détermination des indicateurs, les comparaisons et les croisements, le retraitement des données. Le choix pour cet ouvrage a été d'organiser la réflexion autour d'une unique question, celle de la temporalité dans les méthodes de recherche en didactiques. De nombreux chercheurs de différents pays ont contribué à ce livre, leurs textes ainsi réunis organisent une réflexion méthodologique riche et précise sur cette question.

L'intérêt des chercheurs en didactiques pour la question de la temporalité, traitée sous forme d'interrogations quant aux méthodes de recherche, trouve de multiples raisons : son poids important dans l'élaboration des méthodes de recherche, l'entrecroisement des différentes temporalités dans le champ des didactiques, et enfin l'enjeu actuel de ces questions dans le champ même de la recherche en didactiques.

Les questions de temporalité sont certes importantes pour la plupart des domaines scientifiques, mais elles sont sans doute cruciales pour les différentes didactiques. En effet, en tant que disciplines de recherche fondées sur des recueils de données empiriques portant sur des phénomènes engageant des êtres humains, les didactiques disciplinaires ont à faire inéluctablement avec les temporalités du déroulement de ces phénomènes, du recueil de ces données et de leurs traitements. Nous donnerons ici comme simple exemple l'étude de productions écrites d'élèves, objet que nous pouvons supposer commun à la plupart des didactiques. Ces écrits sont produits selon une chronologie particulière (à un moment donné de l'enseignement, avec des allers-retours, des temps d'arrêts, des accélérations...) qui le plus souvent ne coïncide pas avec celle du document. L'analyse de ces écrits suppose ainsi la prise en compte ou le délaissement de deux temporalités différentes, celle du produit et celle de la production. Cependant, ces problèmes méthodologiques s'amplifient encore par la diversité des temps qui régissent les phénomènes didactiques : temps de l'institution scolaire, temps de l'enseignement, temps des apprentissages, etc. Ces temporalités différentes, inextricablement mêlées, sont des dimensions constitutives des objets d'étude des didacticiens : qu'il s'agisse d'étudier les rapports aux savoirs des élèves, les pratiques des enseignants... les temps qui les formatent et ceux qu'ils génèrent sont à décrire et à interpréter. Ajoutons encore que les chercheurs en didactiques sont confrontés aux temps que construisent les disciplines scientifiques de référence dans les savoirs même qu'elles produisent et qui sont transposés à des fins d'enseignement. Les temps spécifiques de l'histoire, du droit, de la physique pour ne citer que ces exemples, ne sont pas absents des études menées. Enfin, la relative jeunesse de ce domaine scientifique que constitue la recherche en didactiques a pour conséquence une assise des théories et des méthodes développées sans doute plus incertaine que celle que l'on peut éprouver dans des disciplines plus anciennes. La dynamique de construction qui en résulte pèse sur le temps de la recherche. Les différentes temporalités du travail du chercheur posent ainsi des questions spécifiques, par exemple l'absence de normes de référence historiquement établies pour les écrits de ce domaine. Si l'on considère que le croisement des didactiques participe à cette dynamique mêlée d'incertitude, on comprendra que les questions de temporalité aient, périodiquement, agité la communauté des chercheurs de ce domaine. 
Cependant, il nous semble qu'elles apparaissent, à l'heure actuelle, plus vives qu'elles ont pu l'être. Plusieurs raisons peuvent être avancées pour rendre compte de leur acuité. En premier lieu l'état des contraintes institutionnelles actuelles qui pèsent sur les pratiques de recherches, met cette question au cœur de débats importants. L'exigence de cadres temporels précis qui s'accompagne de celle de résultats " rapides" et du cadrage des budgets alloués, questionne la durée des recherches entreprises. À cette circonstance historique s'ajoute celle les difficultés à se faire reconnaître que rencontrent ces disciplines dont nous soulignions plus haut la “ jeunesse ". Les didactiques disciplinaires ont, à l'heure actuelle, délimité plus précisément leurs objets d'étude, elles se sont aujourd'hui explicitement et suffisamment démarquées d'autres disciplines qui portent sur des objets connexes ou qui contribuent aux références théoriques convoquées. Nous pensons par exemple à la psychologie, à la linguistique, à l'épistémologie, etc. Cette prise de distance - dont attestent par ailleurs la tenue et les travaux du séminaire dont nous nous faisons ici l'écho - s'accompagne de reconfigurations à la fois théoriques et méthodologiques. Ainsi, les questions de temporalité, en tant que questions spécifiques aux didactiques, se constituent en lieux pertinents pour penser cette position distanciée. Enfin l'importance accordée à ces questions est peut-être exacerbée par les objets d'étude que privilégient à l'heure actuelle certaines didactiques : l'intérêt pour les pratiques enseignantes, par exemple, a sans doute pesé sur celui accordé à l'étude des différentes temporalités. De même, l'élaboration de récentes méthodes d'analyse (comme celle du synopsis) a relancé le débat sur la prise en compte de ces dernières.

C'est donc en espérant des confrontations et des questionnements fructueux que le thème du second séminaire et celui du présent ouvrage s'est imposé.

Le premier texte de l'ouvrage est de F. Audigier, il constitue une ouverture aux questions de temporalités dans les méthodes de recherche en didactiques par une mise en lumière la tension entre temps subi et temps construit. Cette tension détermine en partie l'activité contrainte du chercheur, qui d'une part subit les temps de l'horloge, de l'école, du contrat de recherche... et qui d'autre part se donne pour tâche de reconstruire et de dévoiler ceux de l'enseignement et des apprentissages, et plus généralement des phénomènes qu'il se donne pour objets de connaissance. Elle a guidé la constitution des chapitres qui suivent ce texte d'ouverture.

Cette tension est tout d'abord spécifiée dans le contexte des recherches "longues ", au cours desquelles le chercheur, simultanément, subit, contrôle et analyse les variations des indicateurs qu'il a choisi pour mener son étude. Un temps " long" du travail empirique de la recherche induit ainsi inéluctablement des modifications dans la population étudiée, dans les positions des sujets etc. qui contraignent la recherche, mais qui la nourrissent aussi par l'étude de ces changements de postures, de ressentis, de savoirs... Trois articles traitent de ces questions particulières et sont regroupés dans un premier chapitre sous le titre "Variations des indicateurs dans une recherche longue ". La dualité temps subi / temps construit est aussi au cœur des questions qui touchent aux découpages temporels effectués dans une recherche en didactiques et aux choix des systèmes d'unités de mesure temporelles. Les problématiques abordées dans ce cadre se distinguent selon que la résolution de cette tension conduit ou non les chercheurs à recourir à plusieurs systèmes concurrents d'unités de temps. Dans le premier cas, les reconstructions des temporalités prises en compte donnent lieu à des systèmes dont il est nécessaire de penser les articulations et les éventuelles contradictions. Les trois articles qui s'inscrivent dans cette problématique sont identifiés comme relevant de " systèmes d'unités de temps non congruentes ", ils constituent le deuxième chapitre de l'ouvrage. Dans le second cas, les unités de temps choisies sont reliées entre elles de manière cohérente par une structure d'échelle, échelle dont la pertinence est à étudier. Le chercheur est alors amené à exhiber des relations entre événements, et à tisser une structure narrative ou explicative des phénomènes qu'il étudie. Les articles qui traitent de cette question figurent dans troisième chapitre intitulé “ problèmes d'échelle ". À la suite de cette déclinaison en questions différenciées, trois textes 
sont présentés dans chapitre conclusif : leur rôle est d'apporter une vision synthétique des questions abordées dans les trois chapitres qui composent le cœur de l'ouvrage.

L'organisation de l'ouvrage permet ainsi des parcours de lecture différents, au cours desquels le lecteur pourra, du moins nous l'espérons, s'emparer de ces questionnements. 